\title{
Absolute measurement of the differential cross section for pp elastic scattering at ANKE-COSY
}

\author{
David Chiladze* for the ANKE Collaboration ${ }^{\dagger}$ \\ High Energy Physics Institute, Tbilisi State University, 0186 Tbilisi, Georgia \\ Institute für Kernphysik, Forschungszentrum Jülich, 52425 Jülich, Germany \\ E-mail: d.chiladzedfz-juelich.de
}

Very little is known experimentally on proton-proton elastic scattering in the energy range from 1.6 to $2.8 \mathrm{GeV}$ for centre-of-mass angles between about 10 and 30 degrees. The differential cross section data that do exist seem to fall systematically below the predictions of the SAID data analysis program. Measurements in this kinematical region are possible at the ANKE spectrometer, which is situated inside the COSY-Jülich storage ring. The fast proton scattered at small angles is registered in the Forward Detection system and the slow recoil proton emerging at large angles is measured by the Silicon Tracking Telescopes.

The ANKE collaboration and the COSY machine crew have jointly developed a very accurate method for determining the absolute luminosity in an experiment at an internal target position. The technique relies on measuring the energy losses due to the electromagnetic interactions of the beam as it passes repeatedly through the thin target and measuring the shift of the revolution frequency by studying the Schottky spectrum. This powerful technique allows to measure the absolute differential cross section for elastic $p p$ scattering with high precision.

First results from this experiment are presented in this contribution.

8th International Conference on Nuclear Physics at Storage Rings-Stori11,

October 9-14, 2011

Laboratori Nazionali di Frascati dell'INFN, Italy

\footnotetext{
*Speaker.

${ }^{\dagger}$ This work has been supported by JCHP FFE, and SRNSF grant 09-1024-4-200.
} 


\section{Introduction}

One of the major projects of the ANKE collaboration, submitted to the COSY PAC in 2005 and 2008 [ [ [], is to contribute to the understanding of the nucleon-nucleon (NN) interaction. NN data are necessary ingredients, not only for the understanding of nuclear forces, but also for the description of meson production and other nuclear reactions at intermediate energies. Therefore any facility that can make a significant and new contribution to this knowledge should do so. This was the underlying philosophy of the very successful COSY-EDDA collaboration [B]].

The complete investigation of the NN interaction needs precise elastic scattering data as input to a phase-shift analysis (PSA), from which the scattering amplitudes at fixed angles can then be reconstructed. A full data set involves experiments with both beam and target particles polarised in the initial state, as well as the determination of the polarisation of one of the final state nucleons [四]. Experiments of this type have been carried out for the $p p$ system up to about $3.0 \mathrm{GeV}$ [5]. The well-known EDDA experiment at COSY has produced a very extensive and important data set of $p p \rightarrow p p$ differential cross sections and the various single and multi-spin observables [目], allowing the construction of reliable isospin $\mathrm{I}=1$ phase shifts up to at least $2 \mathrm{GeV}$ [[]].
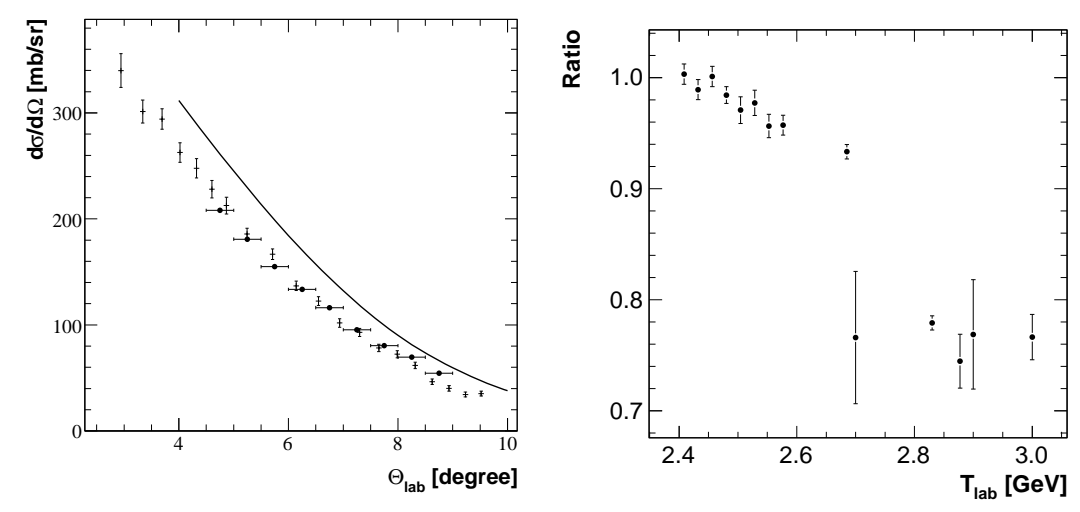

Figure 1: Left: Laboratory differential cross section for elastic proton-proton scattering at 2.65 GeV. The ANKE points, shown by closed circles with bin widths, have systematic uncertainties of $\pm 12 \%$ [8]]. The curve is the SP07 solution from the SAID analysis group [1] and the crosses are experimental data at $2.83 \mathrm{GeV}$ taken from Ref. [9]. Right: Average ratio of experimental data on the $p p$ elastic differential cross section to the SAID predictions [四] for $10^{\circ}<\theta_{c m}<30^{\circ}$ for different energies.

Despite the enormous work undertaken there is still sizable piece of data missing above 1.0 $\mathrm{GeV}$ energy at small scattering angles. Unfortunately lack of data causes severe problems with the small angle SAID predictions above $2.5 \mathrm{GeV}$. These data do not describe well either the ANKE or other published data without introducing an overall scaling factor, but this merely shifts the problem to the larger angle data. Basically, the shapes of the SAID predictions do not seem accurately to reflect reality. As seen from the results shown in the left panel of Fig. W, our data fall about $25 \%$ below the SAID predictions at $2.65 \mathrm{GeV}$ and a similar disagreement is found with the results of Ref. [Q] at the slightly higher energy of $2.83 \mathrm{GeV}$. The variation of the discrepancy with energy is illustrated in the right panel of Fig. I, which shows the ratio of experiment to the SAID prediction averaged, where possible, over the angular range $10^{\circ}<\theta_{c m}<30^{\circ}$. This proves that further precise measurements are required at the upper end of the COSY range, which is also confirmed by the 
disclaimer in the recent SAID update, which states that 'our solution should be considered at best qualitative between 2.5 and $3 \mathrm{GeV}^{\prime}$ [[]].

Fortunately the ANKE spectrometer can provide precise measurement of cross-section and spin observables in the energy range 1.0 to $2.8 \mathrm{GeV}$ for centre-of-mass angles $5^{\circ}<\theta_{c m}<30^{\circ}$. One of the main ingredients in a precise cross-section measurement is the accurate determination of luminosity. This can be achieved using the new method for independent and precise absolute luminosity measurement at an internal experiments at COSY, which was jointly developed by the ANKE collaboration and the COSY machine crew. The technique relies on measuring the energy losses due to the electromagnetic interactions of the beam, as it repeatedly passes through the target, by studying the shift of revolution frequency using the Schottky spectrum [ [ $]$ ].

\section{Experiment Setup}

The COSY COoler SYnchrotron of the Forschungszentrum Jülich is capable of accelerating and storing protons and deuterons with momenta up to $3.7 \mathrm{GeV} / \mathrm{c}$ [ए0]. The ANKE magnetic spectrometer [W] is one of the internal experiments on one of the straight sections of COSY ring. It is designed to study a wide variety of hadronic processes. The circulating beam hits the cluster-jet target [ए2] placed in front of the main spectrometer magnet D2 and ejectiles from the interactions are detected in different detection systems of ANKE (see left panel of Fig. (2)).
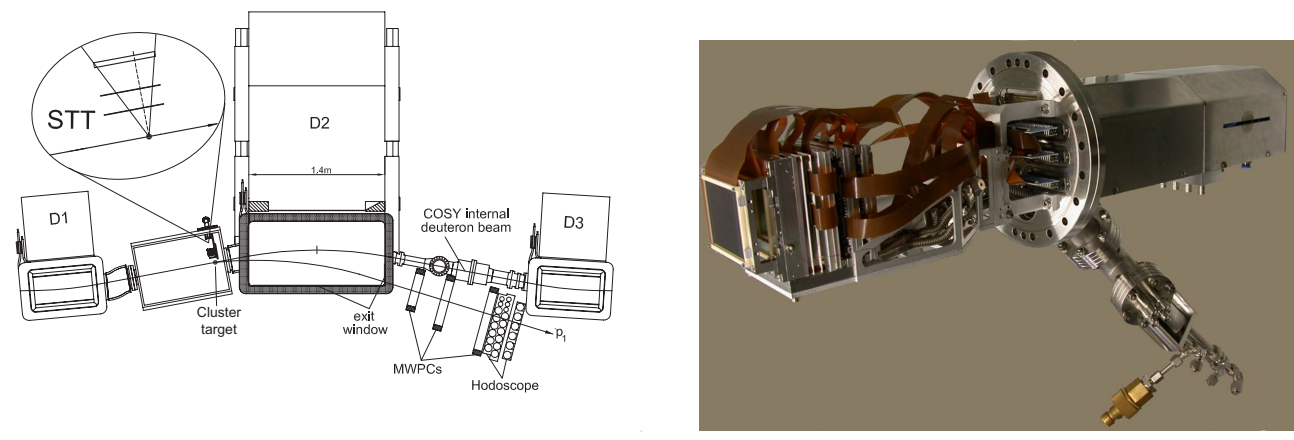

Figure 2: Left: Top view of the ANKE experimental set-up, showing the positions of the three dipole magnets D1, D2, and D3. The hydrogen cluster jet injects target material vertically downwards. The forward detector (FD) consists of three MWPCs and a hodoscope composed of three layers of scintillation counters. Right: Picture of the silicon tracking telescope (STT), consisting of three silicon strip detector layers of different thickness.

Although ANKE contains several detection possibilities only those relevant to current measurements are shown in Fig. \. The forward detector (FD) system consists of three multi-wire chambers for track and momentum reconstruction and two layers of a scintillation hodoscopes for energy-loss determinations and triggering [[1]3]. The silicon tracking telescope (STT), is placed close to the target inside the vacuum chamber, is comprised of three layers of double-sided microstructured silicon strip detectors (right panel of Fig. Z). Measuring the energy loss in the individual layers allows the identification of stopped particles by the $\Delta E / E$ method. The STT, which has self-triggering capabilities, allows one to extend the angular acceptance down to $\theta_{c m} \approx 5^{\circ}$, where the FD has no coverage. 
The experiment was performed using the unpolarised proton beam at eight different energies $T_{p}=1.0,1.6,1.8,2.0,2.2,2.4,2.6,2.8 \mathrm{GeV}$ interacting with a hydrogen cluster-jet target. The $p p$ elastic reaction was identified by the detection of one of the scattered protons in either the FD or in the STT, as well as by the detection of both protons simultaneously. The last option allows a valuable cross-check between these two detection systems and significantly improves the accuracy and reliability of the measurements [14]]. The FD acceptance covers the angular range $10^{\circ}<\theta_{c m}<$ $30^{\circ}$ in the centre-of-mass system. The STT alone can detect protons with an energy higher than 2.5 $\mathrm{MeV}$, which defines the minimum acceptance angle as $\theta_{c m}=5^{\circ}$. Momentum reconstruction with the STT is only possible if the particle is stopped in the third layer. With the current setup of the STT $(70 \mu \mathrm{m}, 300 \mu \mathrm{m}$, and $5 \mathrm{~mm})$ protons with kinetic energies up to $35 \mathrm{MeV}$ can be stopped, which corresponds to around $20^{\circ}$ in the the centre-of-mass system.

The frequency shift measurement of the coasting uncooled proton beam was done with the existing Schottky pick-up and the spectrum analyzer of the stochastic cooling system operated on harmonic number 1000 [ए6]. The statistical distribution of the particles in the beam is at the origin of the Schottky noise. This gives a rise to the current fluctuations which induce a voltage signal at a beam pick-up. The Fourier analysis of the voltage signal, i.e. of the random current fluctuations, by a spectrum analyzer delivers frequency distributions around the harmonics of the revolution frequency. During the $300 \mathrm{~s}$ cycles, the Schottky distribution were recorded in every $10 \mathrm{~s}$ with the $189 \mathrm{~ms}$ sweep time of the analyzer, meaning effectively instantaneous spectra. The mean frequency of the beam was determined from the centroid of the distribution, after subtracting the background noise.

\section{Luminosity Determination}

In order to extract the cross section for any physical reaction, the absolute value of the luminosity must be determined. Luminosity $L$ relates the cross section $\sigma$ of a given physical process to its corresponding event rate $R$ :

$$
R=L \times \sigma
$$

This shows that, apart from a precise measurement of the reaction, it is necessary to determine the luminosity with high accuracy, which is a process-independent quantity and is completely determined by the number of beam and target particles $L=n_{B} \cdot n_{T}$. The measurement of the beam intensity $\left(n_{B}\right)$ is a routine procedure for any accelerator and is performed via the high precision Beam Current Transformer (BCT) devices. For the current experiment, the BCT signal was introduced directly in the data stream and recorded together with the detector information, providing the actual beam intensity at any given moment. Concerning the target density $n_{T}$, as was already mentioned it is determined via the measurement of the beam revolution frequency shift caused by the electromagnetic interaction of the beam and target particles. It should be mentioned that this procedure is only valid if the coasting beam is used without cooling. Details of this method are discussed in Ref. [8], this provides the following formula for the target density determination:

$$
n_{T}=\left(\frac{1+\gamma}{\gamma}\right) \frac{1}{\eta} \frac{1}{(d E / d x) m} \frac{T_{0}}{f_{0}^{2}} \frac{d f}{d t}
$$


Here $T_{0}$ and $f_{0}$ are the initial values of the frequency and energy of the beam, and $\gamma=\left(1-\beta^{2}\right)^{-1 / 2}$ is the Lorentz factor. All three parameters are precisely measured for every experiment at COSY. The stopping power $d E / d x$ and mass $m$ of the target atoms are well known and can be easily obtained ${ }^{1}$. Therefore in order to deduce the absolute values for the target density it is necessary to determine so-called frequency-slip parameter $\eta$ and the beam revolution frequency shift $d f / d t$ during the data taking.
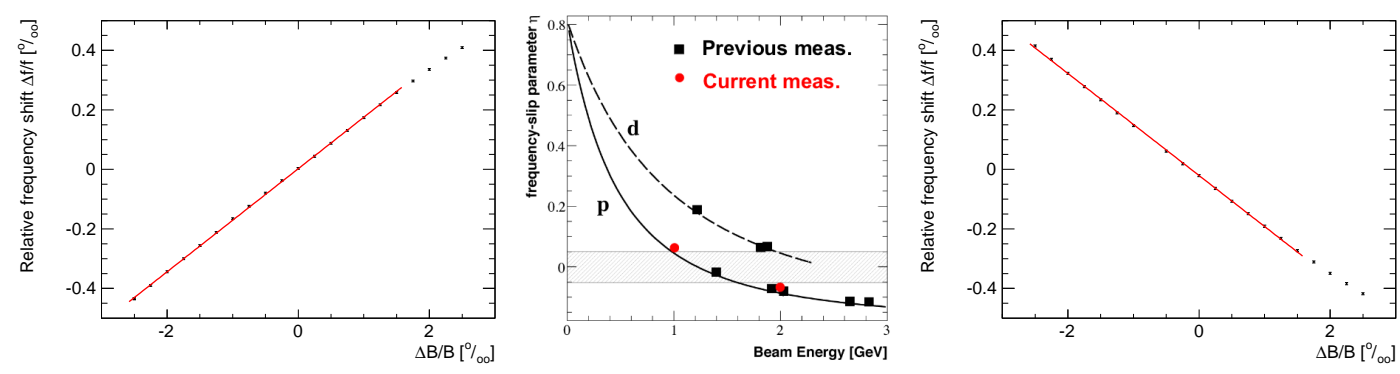

Figure 3: Left and Right: Variation of the mean revolution frequency with the field strength in the bending magnets in parts per thousand for 1.0 and $2.0 \mathrm{GeV}$ beam energy respectively. The slope of the fitted straight line yields the value of the momentum compaction factor $\alpha$ from which the $\eta$ parameter is obtained. Centre: Frequency-slip parameter $\eta$ as a function of the energy of proton and deuteron beams. The experimental points are the results of ANKE measurements during diverse beam runs. These are compared with curves corresponding to the predictions of COSY lattice calculations. The shaded area shows the region with $|\eta|<0.05$ where the error in the energy-loss technique is unacceptably high.

The $\eta$ parameter can be determined using the following relation:

$$
\eta=\frac{1}{\gamma^{2}}-\alpha
$$

where $\alpha$ is a momentum-compaction factor that can be measured by studying the change of revolution frequency due to a change in the magnetic field of the COSY bending magnets [ [ $]$ ]. If the changes are not very large there is a linear relationship between the relative revolution frequency shift $\left(\Delta f / f_{0}\right)$ and the relative change in the field $\left(\Delta B / B_{0}\right)$ expressed in the following form:

$$
\frac{\Delta f}{f_{0}}=\alpha \frac{\Delta B}{B_{0}}
$$

Dedicated cycles were developed to measure the revolution frequency of the beam while changing the magnetic field in the bending magnets by few parts per thousand. Results of this measurement for 1.0 and $2.0 \mathrm{GeV}$ energies are presented on Fig. [], from which the $\eta$ parameter is determined using the eq. (B.3). Final values of frequency-slip parameter for these two energies were $0.0611 \pm 0.0006$ and $-0.0700 \pm 0.0006$ respectively. It is seen that for large values of $\Delta B / B_{0}$ and $\Delta f / f_{0}$ relationship is not linear anymore and requires higher order terms in eq. (B.4). Fortunately this is only relevant if the frequency shift produced by the target is similarly large, which was avoided by limiting the target density in the experiment. Such settings allow one to determine momentum compaction-factor by fitting only the linear part of the $\Delta B / B_{0}$ versus $\Delta f / f_{0}$ relation.

\footnotetext{
${ }^{1}$ The stopping power is available from the database of NIST-PML: http: / / www . nist.gov/pml/data/star
} 
This is confirmed by the good agreement between current values of the $\eta$ parameter and theoretical calculations together with our previous measurements [[]] (see central panel of Fig. [1]). It is important to mention that the $\eta$ parameter has opposite signs for these two energies, which means that the observed frequency shift during the experiment will have different signs. Fig. $\$$ shows how the Schottky spectra developed during the cycle. Distribution shifts to the lower values when for positive $\eta$ and it moves to the higher values when $\eta$ is negative.
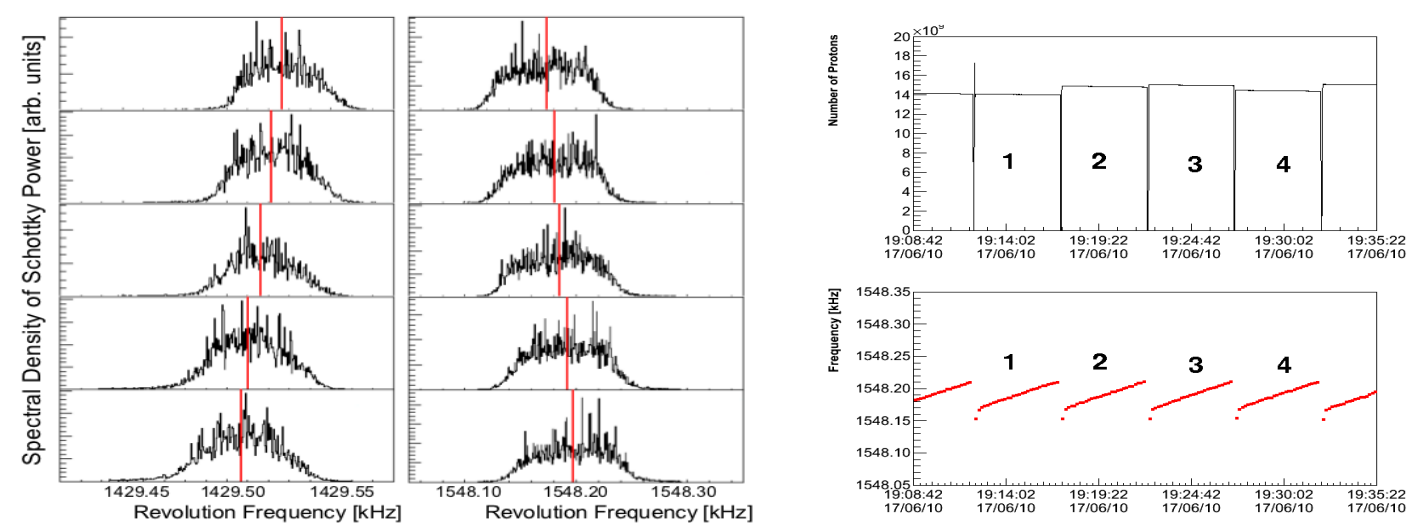

Figure 4: Left: Schottky power spectra obtained during one $300 \mathrm{~s}$ cycle and scaled to harmonic number 1 for 1.0 and $2.0 \mathrm{GeV}$ energy, (left and right column, respectively). Although the data were recorded every 10 seconds, for ease of presentation, only the results from every $70 \mathrm{~s}$ are shown, starting from top to bottom. The mean frequencies are indicated by the vertical lines. Right: The BCT particle current $n_{B}$, and mean revolution frequency $f$ for a sample machine cycle.

For the final target density determination one has to consider that the observed frequency shift is produced not only by the beam-target interaction but also by the residual gas in the COSY ring and its contribution must be subtracted. The measurement of this systematic effect was performed using the dedicated cycles where the ANKE cluster target was on but the beam was steered away from it. This allows to measure frequency shift caused only by the residual gas in the same condition as it is during the normal data taking, which then can be subtracted from the total frequency change (when beam hits the target). Doing background measurements with the target on are necessary because the target produces additional background in the ANKE chamber, which cannot be measured otherwise $[\mathbb{[}]$. The frequency shift caused purely by the target can be presented in this way:

$$
\left(\frac{d f}{d t}\right)_{\text {target }}=\left(\frac{d f}{d t}\right)_{\text {total }}-\left(\frac{d f}{d t}\right)_{b g}
$$

Where $\left(\frac{d f}{d t}\right)_{\text {total }}$ is the observed frequency shift during the usual measurement and $\left(\frac{d f}{d t}\right)_{b g}$ is the frequency shift produced by the residual gas of COSY ring plus the effect from the ANKE cluster target. The right panel of Fig. 目 shows the typical picture of the BCT and revolution frequency during the experiment. The target density is produced for each cycle and the corresponding integrated luminosity determined. Numerical results for the cycles presented in Fig. 1 are listed in

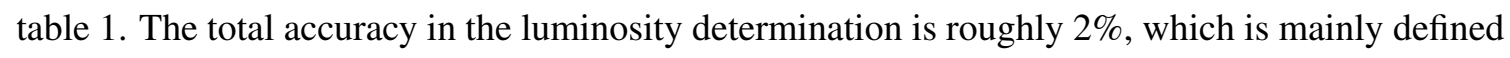
by the error in the measurement of the $\eta$ parameter. 


\begin{tabular}{c|c|c|c} 
Cycle & $d f / d t$ & Target Density & Integrated Luminosity \\
\hline 1 & 0.152 & $2.75 \times 10^{14} \mathrm{~cm}^{-2}$ & $1.67 \times 10^{33} \mathrm{~cm}^{-2}$ \\
2 & 0.151 & $2.74 \times 10^{14} \mathrm{~cm}^{-2}$ & $1.76 \times 10^{33} \mathrm{~cm}^{-2}$ \\
3 & 0.154 & $2.79 \times 10^{14} \mathrm{~cm}^{-2}$ & $1.81 \times 10^{33} \mathrm{~cm}^{-2}$ \\
4 & 0.149 & $2.70 \times 10^{14} \mathrm{~cm}^{-2}$ & $1.68 \times 10^{33} \mathrm{~cm}^{-2}$
\end{tabular}

Table 1: Results of the target density and luminosity measurements for several cycles.

\section{Preliminary Results}

After determining the luminosity it is possible to measure the differential cross-section of $p p$ elastic scattering. For the current work only the data from the forward detector system were analysed. This contains information only about the forward scattered fast particles. The identification of the reaction can be done using the missing-mass technique. Fig. 1 (left panel) shows the missingmass distribution for $T_{p}=2.0 \mathrm{GeV}$ for different angular bins. There is a clear peak on proton mass with no or very little background, which is well separated from the parasitic processes. This provides a clean identification of the reaction. Preliminary results of the differential cross section together with the SAID prediction is presented on the right panel of Fig. [1. The data look very promising, though in the current analysis there are several experimental factors, such as detector efficiency, that are not yet considered. The work is in progress and the final results with total errors of better than $5 \%$ are expected.
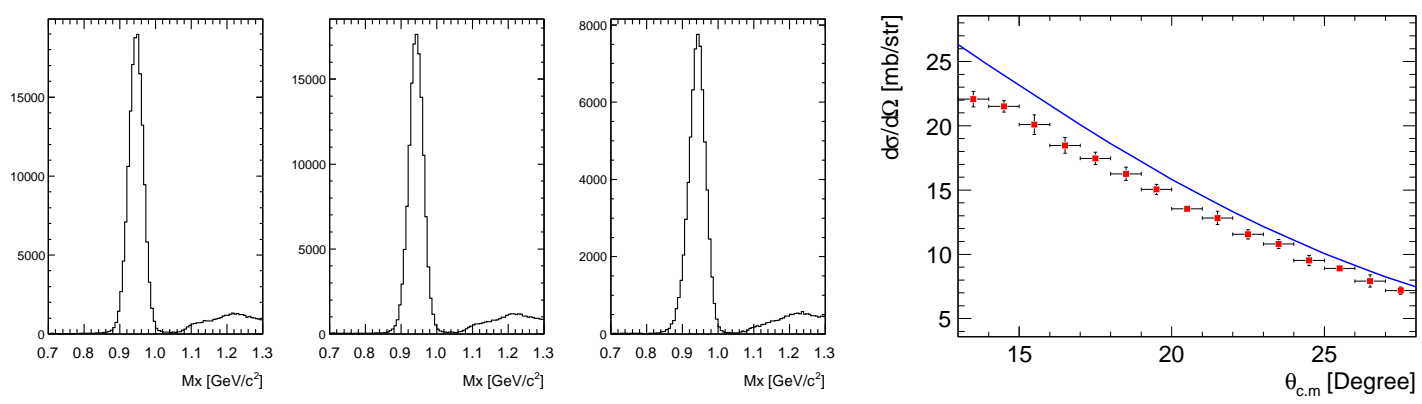

Figure 5: Left: Spectrum of missing masses measured for the $p p \rightarrow p X$ reaction at $2.0 \mathrm{GeV}$ for different angular bins $\left(13-14^{\circ}, 20-21^{\circ}\right.$ and $27-28^{\circ}$ from left to right). The proton peak can be cleanly separated from contributions from the inelastic processes. Right: Preliminary laboratory differential cross section for elastic proton-proton scattering at $2.0 \mathrm{GeV}$. The curve shows SAID 2007 prediction [可].

\section{Conclusions}

First analysis presented in this work shows that ANKE is capable to provide robust $p p$ elastic scattering data in the energy region $T_{p}=1.0-2.8 \mathrm{GeV}$ for the angular range $\theta_{c . m .}=5-30^{\circ}$. Results of this measurement proves that the luminosity can be determined with an accuracy of $2-3 \%$ using the Schottky technique, which gives the possibility to measure $p p$ differential crosssections roughly with $5 \%$ accuracy. 


\section{References}

[1] A. Kacharava, F. Rathmann, and C. Wilkin for the ANKE Collaboration, COSY Proposal \#152, Spin Physics from COSY to FAIR, arXiv:nucl-ex:0511028.

[2] ANKE Collaboration, Status and Future Issues of the Physics Program at COSY with Polarised Hadronic Probes, July 2008; available from http://www.fz-juelich.de/ikp/anke/proposals.shtml.

[3] M. Altmeier et al, Excitation functions of the analyzing power in elastic proton-proton scattering from 0.45 to 2.5 GeV, Eur. Phys. J. A 23, 351 (2005).

[4] J. Bystricky et al., Measurement of np and pp asymmetry with an accelerated polarized deuteron beam from 725 to 1000 MeV per nucleon, Nucl. Phys. A 444, 597 (1985).

[5] R.A. Arndt et al., Updated analysis of NN elastic scattering data to $1.6 \mathrm{GeV}$, Phys. Rev. C 50, 2731 (1994);

R.A. Arndt et al., Nucleon-nucleon elastic scattering analysis to $2.5 \mathrm{GeV}$, Phys. Rev. C 56, 3005, (1997)

R.A. Arndt et al., Nucleon-nucleon elastic scattering to 3 GeV, Phys. Rev. C 62, 034005, (2000)

R.A. Arndt, W.J. Briscoe, R.L. Workman, and I.I. Strakovsky, Interactive on-line program: http://gwdac.phys.gwu.edu/analysis/

[6] M. Altmeier et al., Excitation Functions of the Analyzing Power in p $\vec{p}$ Scattering from 0.45 to 2.5 GeV, Phys. Rev. Lett. 85, 1819 (2000).

[7] NN-online available from http://nn-online.org

[8] H.J. Stein et al., Determination of target thickness and luminosity from beam energy losses, Phys. Rev. ST Accel. Beams 11, 052801 (2008).

[9] I. Ambats et al., Systematic study of $\pi^{ \pm} p, K^{ \pm} p, p p$, and $\bar{p} p$ forward elastic scattering from 3 to 6 GeV/c, Phys. Rev. D 9, 1179 (1974).

[10] R. Maier et al., Cooler synchrotron COSY - Performance and perspectives, Nucl. Instrum. Methods A 390, 1 (1997).

[11] S. Barsov et al., ANKE, a new facility for medium energy hadron physics at COSY-Jülich, Nucl. Instr. Meth. A 462, 364 (2001).

[12] A. Khoukaz et al., Systematic studies on hydrogen cluster beam production, Eur. Phys. J. D 5, 275 (1999).

[13] S. Dymov et al., The Forward Detector of the ANKE Spectrometer. Tracking System and Its Use in Data Analysis, Part. Nucl. Lett. 1, 40 (2004).

[14] D. Chiladze et al., Determination of deuteron beam polarizations at COSY, Phys. Rev. ST Accel. Beams, 9, 050101 (2006).

[15] R. Schleichert et al., A self-triggering silicon-tracking telescope for spectator proton detection, IEEE Trans. Nucl. Sci. 50, 301 (2003).

[16] D. Prasuhn et al., Electron and stochastic cooling at COSY, Nucl. Instrum. Methods Phys. Res. A 441, 167 (2000). 